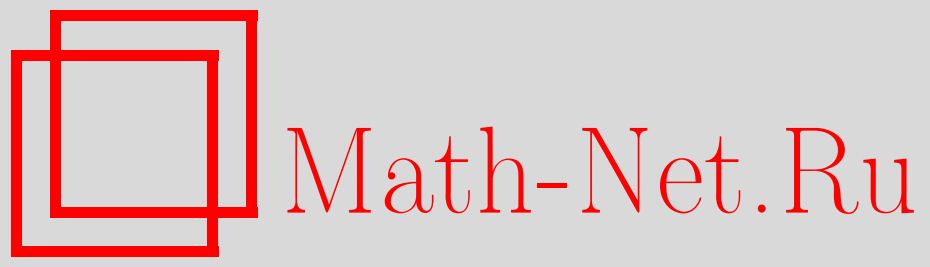

А. Черноуцан, Относительность движения в задачах кинематики, Квант, 2019, номер 2, 43-50

DOI: https://doi.org/10.4213/kvant20190205

Использование Общероссийского математического портала Math-Net.Ru подразумевает, что вы прочитали и согласны с пользовательским соглашением http://www . mathnet.ru/rus/agreement

Параметры загрузки:

IP : 3.91 .87 .62

26 апреля 2023 г., 18:16:26

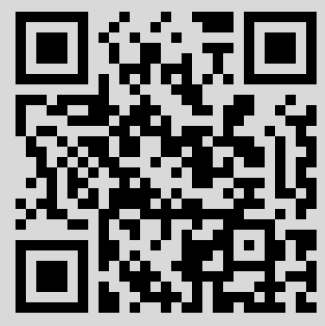




\section{ОтносИтеЛьность движения в задачах кинематики}

\section{А. ЧЕРНОУЦАН}

$\Pi$ РИ РЕШЕНИИ ЗАДАЧ МЕХАНИКИ ПОлезно владеть умением переходить в движущиеся системы отсчета (CO). Выбор удачной $\mathrm{CO}$ может привести к заметному упрощению решения, сделать трудные моменты наглядными и очевидными. В некоторых задачах условие сразу содержит данные, относящиеся к различным $\mathrm{CO}$, и использование формул перехода от одной СО к другой становится неизбежным. Это относится, например, к движению летательных аппаратов (самолетов) при наличии ветра или к движению плавательных средств (лодок, катеров) при наличии течения. Летчик управляет скоростью самолета относительно воздуха (в $\mathrm{CO}$, связанной с движущемся воздухом): в режиме прямолинейного движения она направлена вдоль линии корпуса самолета, а ее величина определяется выбранной летчиком мощностью. Точно так же капитан управляет движением катера относительно воды. Для расчета движения самолета относительно земли (в этой СО он должен лететь в заданном направлении, от пункта отправления к пункту назначения, а от модуля скорости относительно земли зависит время полета) или катера относительно берега нужно применить закон сложения скоростей.

Закон сложения скоростей связывает между собой скорость $\vec{v}_{2}$ тела относительно земли (неподвижной СО), его скорость $\vec{v}_{21}$ относительно движущегося тела (т.е. относительно поступательно движущейся СО, связанной с движущимся телом 1) и скорость $\vec{v}_{1}$ самой СО (скорость тела 1):

$$
\vec{v}_{2}=\vec{v}_{21}+\vec{v}_{1} .
$$

DOI: https://doi.org/10.4213/kvant20190205
(В задачах удобно применять не численные, a «говорящие» буквенные индексы.) Отметим, что поскольку речь пока идет о постyпательно движущихся СО, то можно говорить о скорости $\mathrm{CO}$, не указывая, о какой ее точке идет речь. В случае вращающихся $\mathrm{CO}$ под $\vec{v}_{1}$ будет подразумеваться скорость той точки СО, где в данный момент находится тело (ее называют переносной скоростью). Аналогично записываются формулы для сложения перемещений и сложения ускорений (для поступательно движущихся СО):

$$
\vec{s}_{2}=\vec{s}_{21}+\vec{s}_{1}, \vec{a}_{2}=\vec{a}_{21}+\vec{a}_{1} .
$$

Во многих задачах формулы (1), (2) удобно применять в виде, сразу выражающем относительную скорость (перемещение, ускорение) одного движущегося тела относительно другого (относительно поступательно движущейся СО, связанной с этим другим телом):

$$
\vec{v}_{21}=\vec{v}_{2}-\vec{v}_{1}, \vec{s}_{21}=\vec{s}_{2}-\vec{s}_{1}, \quad \vec{a}_{21}=\vec{a}_{2}-\vec{a}_{1} .
$$

(Формулы в таком виде удобны для запоминания ввиду повторения индексов в правой и левой частях).

При решении математических задач на движение, например катера по воде, формула (1) используется уже в младших классах в виде простого правила: при движении вниз по течению скорость течения надо прибавлять, против течения - вычитать. Объясняют это правило интуитивно просто: сначала катер перемещается относительно воды, а потом вместе с водой. Результат такой же, как если бы все происходило одновременно. Но такое же рассуждение годится для объяснения и общей векторной формулы (1): сначала тело 2 перемещается относительно $\mathrm{CO}_{1}$ на $\vec{v}_{21} \Delta t$ (тело 1 при этом неподвижно), а затем вместе с телом 1 и $\mathrm{CO}_{1}-$ на $\vec{v}_{1} \Delta t$. В итоге все на своих местах - и тело 2 и $\mathrm{CO}_{1}$ !

В случае движения всех тел вдоль одной прямой формулы (1)-(3) удобно сразу применять в проекции на выбранную ось.

Задача 1. Скорость моточиклиста $v_{\mathrm{M}}=54 \kappa \mathrm{\kappa} /$, а скорость встречного ветра $v_{\mathrm{B}}=3 \mathrm{\kappa м} / \mathrm{c}$. Какова скорость ветра в системе отсчета, связанной с мотоциклистом? В ответе дайте модуль скорости.

Решение. Удобно воспользоваться формулой (3):

$$
\vec{v}_{\mathrm{BM}}=\vec{v}_{\mathrm{B}}-\vec{v}_{\mathrm{M}}
$$


и записать ее в проекции на ось $x$, направленную вдоль скорости мотоциклиста:

$$
v_{\text {Вм } x}=v_{\text {в } x}-v_{\text {м } x}=\left(-v_{\text {в }}\right)-v_{\text {м }}=-18 \mathrm{M} / \mathrm{c} .
$$

Если выбрать ось «по ветру», то проекция получится положительной:

$$
v_{\text {вм } x}=v_{\text {в }}-\left(-v_{\text {м }}\right)=18 \mathrm{~m} / \mathrm{c} \text {. }
$$

Следующие несколько задач относятся к упомянутым выше задачам навигации - управлением летательными или плавательными аппаратами в движущихся средах.

Задача 2. Катер, переправляясь через реку шириной $L=800 \mathrm{~m}$, двигался со скоростью $v_{\text {кв }}=4 \mathrm{~m} / \mathrm{c}$ перпендикулярно течению реки в системе отсчета, связанной с водой. На сколько будет снесен катер течением, если скорость течения реки $v_{\mathrm{B}}=1,5 \mathrm{~m} / \mathrm{c}$ ?

Решение. Если векторы скоростей не параллельны, то надо начать с векторной записи закона сложения скоростей, затем сделать рисунок, соответствующий этому векторному равенству, и только потом, опираясь на этот рисунок, проводить вычисления. В данной задаче, согласно формуле (1),

$$
\vec{v}_{\mathrm{K}}=\vec{v}_{\text {Кв }}+\vec{v}_{\text {в }} .
$$

Соответствующий этому равенству векторный треугольник изображен на рисунке 1 с

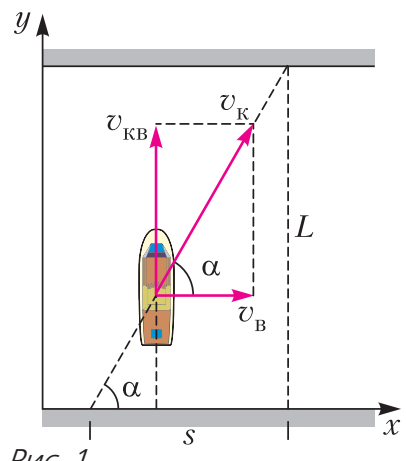

Pис. 1

учетом того, что скорость воды $\vec{v}_{\text {в }}$ параллельна берегу, а скорость катера относительно воды $\vec{v}_{\text {кв }}$ перпендикулярна берегу. Угол $\alpha$, который траектория катера составляет с берегом, равен углу в треугольнике скоростей. Для искомого расстояния $s$ получаем

$$
s=L \operatorname{ctg} \alpha=L \frac{v_{\mathrm{B}}}{v_{\text {Кв }}}=300 \mathrm{M} .
$$

Можно было не вводить угол $\alpha$ и воспользоваться подобием треугольников. А еще можно, как при рассмотрении броска под углом, записать закон движения в проекциях на оси $x$ и $y$. Из закона сложения скоростей следует, что $v_{\text {к } x}=v_{\text {в }}$, а $v_{\text {кy }}=v_{\text {кв }}$. Получаем два равномерных движения:

$$
s=v_{\mathrm{B}} t, L=v_{\mathrm{KB}} t .
$$

Исключая $t$, находим $s$.

Задача 3. Самолет летел на север со скоростью $v_{\mathrm{c} 1}=48 \mathrm{~m} /$ с относительно земли. С какой скоростью относительно земли будет лететь самолет, если подует западный ветер со скоростью $v_{\mathrm{B}}=14 \mathrm{~m} / \mathrm{c}$ ?

Решение. Пока самолет летел в отсутствие ветра, его скорости относительно земли и относительно воздуха совпадали и были направлены на север: $\vec{v}_{\text {c1 }}=\vec{v}_{\text {св }}$. Что же изменилось после того, как подул западный ветер? Судя по условию задачи, летчик пока не знает, что подул ветер, и ничего не сделал для корректировки полета. Это значит, что корпус самолета все так же направлен на север и мотор работает с прежней мощностью. Это, в свою очередь, означает, что относительно воздуха самолет движется с той же скоростью $\vec{v}_{\text {св }}$, равной 48 м/с и направленной на север. А вот новую скорость самолета относительно земли найдем из закона сложения скоростей:

$$
\vec{v}_{\mathrm{c} 2}=\vec{v}_{\mathrm{cв}}+\vec{v}_{\mathrm{B}} .
$$

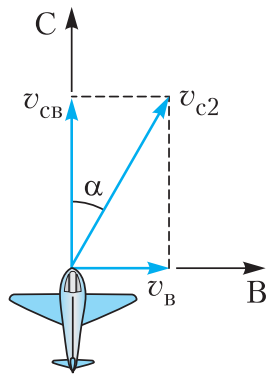

Pис. 2

Изобразив треугольник скоростей (рис.2), для величины искомой скорости получим

$$
v_{\mathrm{c} 2}=\sqrt{v_{\mathrm{CB}}^{2}+v_{\mathrm{B}}^{2}}=50 \mathrm{M} / \mathrm{c} .
$$

Нетрудно также найти угол отклонения самолета от курса на север:

$$
\sin \alpha=\frac{v_{\text {в }}}{v_{\text {c2 }}}=0,28, \text { и } \alpha \approx 16^{\circ} .
$$

Задача 4. При переправе через реку шириной $L=80$ м надо попасть в точку, лежашую на $s=60$ м выше по течению, чем точка старта. Лодочник управляет моторной лодкой так, что она движется точно киели со скоростью $v_{\text {л }}=4,5 \mathrm{M} / \mathrm{c}$ относительно берега. Какова при этом скорость лодки относительно водь, если скорость течения реки $v_{\mathrm{B}}=2,1 \mathrm{~m} / \mathrm{c}$ ? 
Решение. Скорость лодки относительно берега $\vec{v}_{л}$ направлена вдоль указанной в условии линии движения (рис.3), а скорость

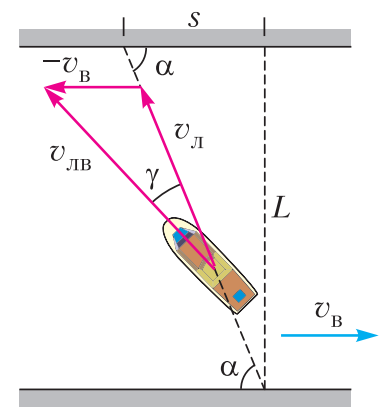

Pис. 3

воды равна скорости течения и направлена параллельно берегу. Скорость лодки, которой управляет лодочник, относительно воды выражается формулой (3):

$$
\vec{v}_{\text {лв }}=\vec{v}_{\text {л }}-\vec{v}_{\text {В }}=\vec{v}_{\text {л }}+\left(-\vec{v}_{\mathrm{B}}\right) .
$$

Это векторное равенство изображено на рисунке 3. Величину этой относительной скорости найдем с помощью теоремы косинусов:

$$
\begin{aligned}
v_{\text {лв }}=\sqrt{v_{\pi}^{2}+v_{\mathrm{B}}^{2}-2 v_{л} v_{\mathrm{B}} \cos \left(180^{\circ}-\alpha\right)}= \\
=\sqrt{v_{\pi}^{2}+v_{\mathrm{B}}^{2}+2 v_{\pi} v_{\mathrm{B}} \cos \alpha},
\end{aligned}
$$

где $\alpha-$ угол между траекторией лодки и берегом. Косинус этого угла найдем из треугольника расстояний:

$$
\cos \alpha=\frac{s}{\sqrt{s^{2}+L^{2}}}=0,6 .
$$

Подставляя числа, получаем

$$
v_{\text {лв }}=6 \mathrm{M} / \mathrm{c} \text {. }
$$

Можем также найти угол $\gamma$, на который лодочник должен отклонить нос лодки от заданной траектории. Для этого придется воспользоваться теоремой синусов:

$\frac{\sin \gamma}{v_{\text {в }}}=\frac{\sin \alpha}{v_{\text {лв }}}$, откуда $\sin \gamma=0,28$, и $\gamma \approx 16^{\circ}$.

Задача 5. В безветренную погоду самолет затрачивает на перелет между городами $t_{1}=6$ ч. На сколько минут увеличится время полета, если будет дуть боковой

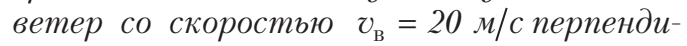
кулярно линии полета? Скорость самолета относительно воздуха равна $v_{\mathrm{cB}}=328 \mathrm{\kappa m} / u$.
Решение. В первом случае самолет перемещается относительно земли со скоростью $\vec{v}_{\text {св }}$, т.е. расстояние между городами связано с временем полета формулой

$$
s=v_{\mathrm{cB}} t_{1} .
$$

Если дует боковой ветер, то, для того чтобы сохранить движение по прежней прямой линии, соединяющей города $A$ и $B$, летчику надо отклонить нос самолета в сторону ветра (рис.4; вид сверху). Вдоль оси самолета

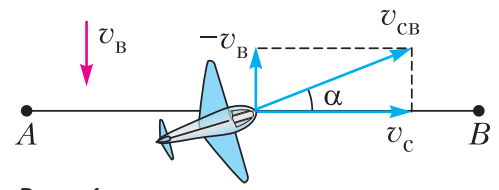

Pис. 4

будет направлена скорость самолета относительно воздуха, которая связана со скоростью самолета относительно земли и скоростью воздуха (ветра) формулой (3):

$$
\vec{v}_{\text {св }}=\vec{v}_{\mathrm{c}}-\vec{v}_{\mathrm{B}}=\vec{v}_{\mathrm{c}}+\left(-\vec{v}_{\mathrm{B}}\right) .
$$

Из рисунка 4, на котором изображено это векторное равенство, находим величину скорости самолета относительно земли:

$$
v_{\mathrm{c}}=\sqrt{v_{\mathrm{cB}}^{2}-v_{\mathrm{B}}^{2}},
$$

после чего получаем новое время полета:

$$
t_{2}=\frac{s}{v_{\mathrm{c}}}=\frac{v_{\mathrm{cB}} t_{1}}{\sqrt{v_{\mathrm{cB}}^{2}-v_{\mathrm{B}}^{2}}}=369 \text { мин . }
$$

Это на 9 минут больше, чем в отсутствие ветра. Напоследок поможем летчику вычислить необходимый угол $\alpha$ отклонения корпуса самолета от линии полета:

$$
\sin \alpha=\frac{v_{\text {в }}}{v_{\text {св }}}=0,22, \text { и } \alpha=12,7^{\circ} .
$$

Следующая задача демонстрирует, как можно с помощью закона сложения скоростей графически решать задачи, связанные с нахождением экстремума.

Задача 6. Скорость течения реки $v_{\mathrm{B}}=5 \mathrm{~m} / \mathrm{c}$, ее ширина $L=32 \mathrm{м}$. Переправляясь через реку на лодке, скорость которой относительно воды $v_{л в}=4 \mathrm{M} / \mathrm{c}$, рулевой обеспечил наименьший возможный снос лодки течением. Чему равен этот снос?

Решение. Расширим рамки задачи - найдем минимальный снос для различных значений скорости лодки относительно воды. 
Если $v_{\text {лв }}>v_{\text {в }}$, то лодочник может выбрать такой курс (т.е. так направить $\vec{v}_{\text {лв}}$ ), что снос лодки будет равен нулю. На рисунке 5, $a$ изображен закон сложения скоростей

$$
\vec{v}_{\text {л }}=\vec{v}_{\text {лв }}+\overrightarrow{\vec{v}}_{\text {в }}
$$

для случая, когда $\vec{v}_{\text {л }}$ перпендикулярна ли-

a)

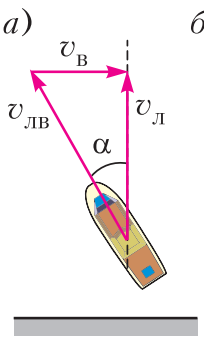

PUC. 5

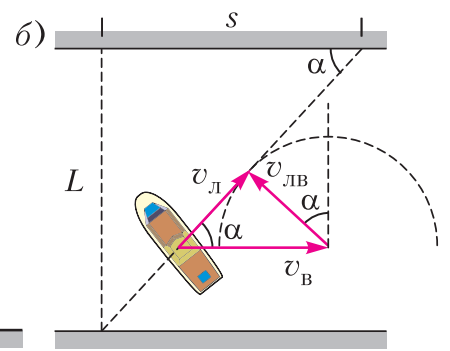

нии берега. Корпус лодки должен при этом составлять с перпендикулярным направлением угол, синус которого равен

$$
\sin \alpha=\frac{v_{\mathrm{B}}}{v_{\text {лв }}} .
$$

Если же $v_{\text {лв }}<v_{\text {в }}$ (как в нашем случае), то снос нельзя сделать равным нулю и надо выбрать такое направление скорости $\vec{v}_{\text {лв }}$, при котором скорость $\vec{v}_{\text {л }}$ будет составлять максимальный угол с линией берега. На рисунке 5,б изображен закон сложения скоростей для этого случая. К концу постоянного вектора $\vec{v}_{\text {в }}$ приставляется вектор меньшей длины $\vec{v}_{\text {лв }}$, который может принимать все возможные направления «от берега». Концы всех этих векторов описывают полуокружность радиусом $v_{\text {лв }}$, а наибольший угол $\alpha$, который вектор $\vec{v}_{\text {л }}$ может составлять с линией берега, соответствует случаю, когда линия скорости $\vec{v}_{\text {л }}$ проходит по касательной к окружности. Из рисунка находим

$$
\sin \alpha=\frac{v_{\text {лв }}}{v_{\text {в }}}=0,8 .
$$

(Такой же угол составляет при этом $\vec{v}_{\text {лв }}$, т.е. направление корпуса лодки, с перпендикуляром к линии берега. Отметим, что корпус лодки при этом направлен перпендикулярно вектору $\vec{v}_{\text {л }}$, т.е. линии движения лодки.) Минимальный снос при этом равен

$$
s=L \operatorname{ctg} \alpha=24 \text { м. }
$$

В следующих двух задачах мы будем наблюдать за падением капель дождя.
Задача 7. Когда автобус стоит на остановке, капли дождя оставляют на боковом стекле вертикальные следы, а когда он едет со скоростью $v_{\mathrm{a}}=72 \kappa \mathrm{k} / u$, следь капель наклонены к вертикали под углом $\alpha=30^{\circ}$. С какой скоростью падают капли дождя?

Решение. Когда в задаче идет речь о падении капель, у учеников часто возникает вопрос: как можно говорить о скорости падения капель, когда все они падают с ускорением $g$ и достигают земли с разной скоростью? Это не так. Каждая капля достигает поверхности земли уже в режиме установившегося движения, падая с такой скоростью, при которой сила сопротивления воздуха уравновешивает силу тяжести (в отсутствие ветра). Как показывает анализ, крупные капли падают с большей скоростью, чем мелкие. В задачах обычно предполагается, что все капли данного дождя имеют одинаковую массу («монодождь»), т.е. падают с одной и той же скоростью.

Первая фраза условия означает, что ветер отсутствует (об учете ветра речь пойдет в следующей задаче). В СО, связанной с движущимся автобусом, капли дождя падают под углом $\alpha$ к вертикали. Запишем закон сложения скоростей в форме (3):

$$
\vec{v}_{\text {ка }}=\vec{v}_{\mathrm{K}}-\vec{v}_{\mathrm{a}}
$$

и учтем (рис.6), что скорость капель направлена вертикально, а скорость автобуса - горизонтально. Получим

$$
v_{\mathrm{K}}=v_{\mathrm{a}} \operatorname{ctg} \alpha \approx 34 \mathrm{M} / \mathrm{c} .
$$

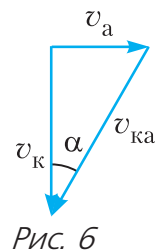

Pис. 6
Задача 8. При скорости ветра, равной $v_{\mathrm{B} 1}=10 \mathrm{~m} / \mathrm{c}$, капли дождя падают под углом $\alpha_{1}=30^{\circ} \kappa$ вертикали. При какой скорости ветра капли будут падать под углом $\alpha_{2}=60^{\circ} \kappa$ вертикали?

Решение. В СО, движущейся со скоростью ветра, воздух неподвижен, т.е. капли дождя падают вертикально вниз. Скорость $v_{\text {кв }}$ падения капель в этой СО зависит только от размеров капель и не зависит от скорости ветра. Записав и нарисовав (рис.7) закон сложения скоростей для первого ветра:

$$
\vec{v}_{\mathrm{K} 1}=\vec{v}_{\mathrm{KB}}+\vec{v}_{\mathrm{B} 1},
$$

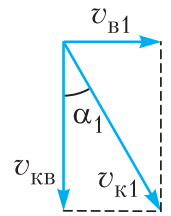

Pис. 7 
придем к соотношению

$$
\operatorname{tg} \alpha_{1}=\frac{v_{\mathrm{B} 1}}{v_{\mathrm{\kappa в}}} .
$$

Аналогичное соотношение получается для второго ветра. Исключив $v_{\text {кв }}$, получим

$$
v_{\mathrm{B} 2}=v_{\mathrm{B} 1} \frac{\operatorname{tg} \alpha_{2}}{\operatorname{tg} \alpha_{1}}=30 \mathrm{~m} / \mathrm{c} \text {. }
$$

В следующей задаче удается учесть взаимодействие мяча с движущимся воздухом с помощью перехода в другую СО.

Задача 9. После удара футболиста мяч полетел в сторону ворот, находящихся на расстоянии $L=32 \mathrm{м}$, со скоростью $v_{0}=$ $=25 \mathrm{~m} / \mathrm{c}$ под углом $\alpha$ к горизонту таким, что $\cos \alpha=0,8$. Из-за бокового ветра, дующего вдоль ворот перпендикулярно начальной скорости мяча, горизонтальное смещение мяча в плоскости ворот оказалось равным $s=2$ м. Найдите время полета мяча до плоскости ворот. Мяч не врашается, скорость ветра $u=10 \mathrm{~m} / \mathrm{c}$.

Решение. В СО земли траектория мяча сложная пространственная кривая (рис.8, $a$; вид сверху; буква «Ц» обозначает центр ворот). В результате воздействия бокового

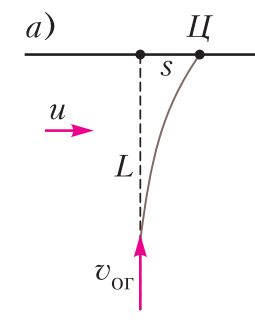

Pис. 8

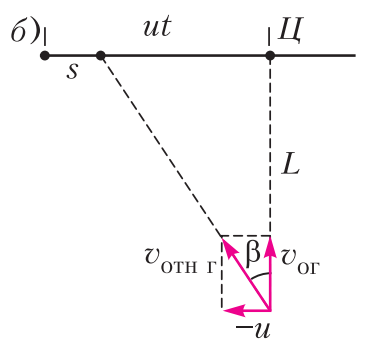

CO воздуха ветра место пересечения мячом линии ворот смещено на $s$ вправо, в сторону ветра. Однако в СО, связанной с воздухом, сила сопротивления направлена против скорости и движение происходит в вертикальной плоскости, направление которой задается горизонтальной составляющей начальной относительной скорости (рис.8,б; вид сверху)

$$
\vec{v}_{\text {Отн г }}=\vec{v}_{0 \text { г }}-\vec{u}=\vec{v}_{0 \text { г }}+(-\vec{u}) .
$$

Перемещаясь в этой плоскости, мяч сместится влево на

$$
L \operatorname{tg} \beta=L \frac{u}{v_{0 г}}=L \frac{u}{v_{0} \cos \alpha} .
$$

Все время полета центр ворот двигался в этой СО влево со скоростью $u$, переместившись на расстояние ut. Однако в момент пересечения мячом линии ворот он должен находиться на $s$ правее центра ворот (см. рис. $8, a)$. Получаем

$$
s=u t-\frac{L u}{v_{0} \cos \alpha}
$$

откуда

$$
t=\frac{s}{u}+\frac{L}{v_{0} \cos \alpha}=1,8 \mathrm{c}
$$

В рамках кинематики можно на равных правах использовать СО, движущиеся не только равномерно, но и ускоренно (в динамике необходимо различать инерциальные и неинерциальные СО). Проиллюстрируем это следующим примером.

Задача 10. Скоростной лифт опускается с ускорением $a=5 \mathrm{~m} / \mathrm{c}^{2}$ относительно земли. В некоторый момент времени с потолка лифта начинает падать болт. Высота лифта $h=2,5$ м. Определите время падения болта.

Решение. Можно, конечно, решать эту задачу в СО земли. Надо записать законы движения болта и дна лифта (с учетом их скорости в момент отрыва) и приравнять их координаты (условие падения). Однако в СО лифта болт падает с нулевой начальной скоростью с ускорением

$$
a_{\text {бл }}=a_{6}-a_{\text {лI }}=g-a
$$

(ось направлена вниз) и пролетает расстояние $h$. Получаем

$$
h=\frac{(g-a) t^{2}}{2}, \text { и } t=\sqrt{\frac{2 h}{g-a}}=1 \mathrm{c} .
$$

Следующую задачу удобно решать переходом в СО одного из тел, которое движется с ускорением.

Задача 11. Два тела начинают одновременно двигаться по прямой навстречу друг другу с начальными скоростями $v_{10}=10 \mathrm{~m} / \mathrm{c}$ и $v_{20}=20 \mathrm{~m} / \mathrm{c}$ ис постоянньми ускорениями $a_{1}=2 \mathrm{~m} / c^{2} u a_{2}=1 \mathrm{~m} / \mathrm{c}^{2}$, направленными противоположно соответствуюшим начальным скоростям. Начальное расстояние между телами $s_{0}=200 \mathrm{M}$. Определите минимальное расстояние между телами. 
Решение. Стандартное решение этой задачи выглядит несложно: надо записать законы движения двух тел, выразить расстояние между ними в зависимости от времени и найти минимум полученного выражения. Однако если перейти в СО одного из тел, то другое тело будет приближаться к нему с начальной скоростью $v_{0}=v_{10}+v_{20}$ и с постоянным ускорением $a=a_{1}+a_{2}$, направленным против начальной скорости. Из стандартной формулы кинематики

$$
0-v_{0}^{2}=-2 a s
$$

находим, что это другое тело пройдет до разворота расстояние

$$
s=\frac{v_{0}^{2}}{2 a}=\frac{\left(v_{10}+v_{20}\right)^{2}}{2\left(a_{1}+a_{2}\right)}=150 \mathrm{~m},
$$

т.е. минимальное расстояние между телами составляет

$$
l_{\text {min }}=s_{0}-s=50 \mathrm{M} .
$$

Следующие три задачи возвращают нас к теме графического нахождения экстремума.

Задача 12. На ленту транспортера, ползущую со скоростью $v_{л}=4 \mathrm{~m} / \mathrm{c}$, сбоку сталкивают коробку. Скорость коробки сразу после попадания на ленту равна $v_{\mathrm{\kappa} 0}=3 \mathrm{~m} / \mathrm{c}$ и перпендикулярна скорости ленты. Какую минимальную скорость относительно земли будет иметь коробка во время движения? Сила трения достаточна велика, так что коробка не соскальзывает с ленты.

Решение. В СО, связанной с землей, движение коробки выглядит сложно. Ясно, что начальная скорость коробки равна $v_{\kappa}$, а конечная равна $v_{л}$. В процессе движения скорость изменяет величину и направление, поворачиваясь на $90^{\circ}$. Но как при этом меняется скорость коробки - непонятно. Однако в СО, связанной с движущейся лентой транспортера, движение коробки выглядит совсем просто. По неподвижной

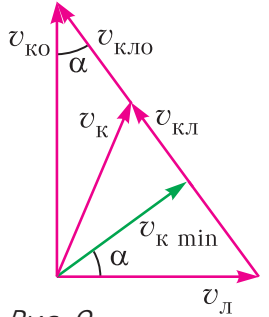

Pис. 9 ленте начинает двигаться коробка с начальной скоростью

$$
\vec{v}_{\text {Кл } 0}=\vec{v}_{\text {К } 0}-\vec{v}_{\text {л }}
$$

и продолжает двигаться по этой прямой с уменьшающейся (за счет трения) скоростью. Чтобы найти скорость коробки относительно земли в произвольный момент времени, надо еще раз применить закон сложения скоростей (рис.9):

$$
\vec{v}_{\text {К }}=\vec{v}_{\text {Кл }}+\vec{v}_{л} .
$$

Как видно из рисунка, скорость коробки принимает наименьшее значение в тот мо-

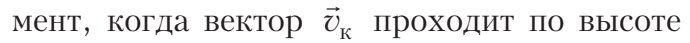
треугольника скоростей. Получаем

$$
v_{\text {К } \min }=v_{\text {К } 0} \sin \alpha=v_{\text {К } 0} \frac{v_{\text {Л }}}{\sqrt{v_{\mathrm{K} 0}^{2}+v_{\pi}^{2}}}=2,4 \mathrm{~m} / \mathrm{c} \text {. }
$$

Задача 13. Автомобиль приближается к пункту $A$ со скоростью $v_{\mathrm{a}}=80 \kappa \mathrm{k} / \mathrm{u} . B$ тот момент, когда ему оставалось проехать $s_{0}=10 \kappa м$, из пункта А в перпендикулярном направлении выезжает грузовик со скоростью $v_{г}=60 \kappa м / u$. Чему равно наименьшее расстояние (в км) между автомобилем и грузовиком?

Решение. Конечно, эту задачу можно решить «в лоб» чисто алгебраически, не переходя в другую СО. Для этого надо выразить расстояние между автомобилем и грузовиком как функцию времени и исследовать полученное выражение на минимум. Проделайте эти вычисления и сравните с тем решением, которое предлагаем мы.

Когда речь идет об изучении взаимного расположения двух движущихся тел, задача, как правило, существенно упрощается при переходе в СО, связанную с одним из этих тел. (В этой СО надо рассматривать только одно движущееся тело.) Перейдем в СО автомобиля. В этой СО грузовик движется с постоянной скоростью

$$
\vec{v}_{\text {га }}=\vec{v}_{\text {г }}-\vec{v}_{\mathrm{a}}=\vec{v}_{\text {г }}+\left(-\vec{v}_{\mathrm{a}}\right)
$$

по прямой линии мимо неподвижного автомобиля (рис.10). Наименьшее расстояние

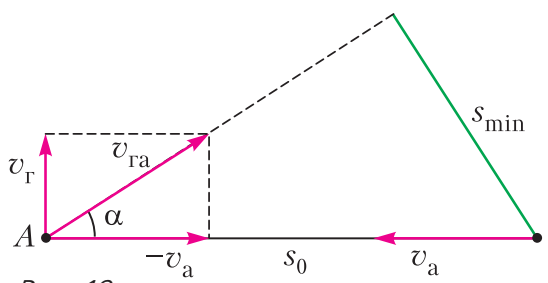

Рис. 10

между ними находится построением - оно равно длине перпендикуляра, опущенного на эту линию:

$$
s_{\min }=s_{0} \sin \alpha=s_{0} \frac{v_{\Gamma}}{v_{\text {га }}}=s_{0} \frac{v_{\text {Г }}}{\sqrt{v_{\text {г }}^{2}+v_{\mathrm{a}}^{2}}}=6 \text { км. }
$$


Аналогичные рассуждения проходят и в случае свободного движения двух тел в поле тяжести, хотя движение каждого тела в отдельности выглядит сложнее.

Задача 14. Два камня расположены на одной горизонтали на расстоянии $s_{0}=42 \mathrm{м}$ друг от друга. Один камень бросают вертикально вверх со скоростью $v_{10}=5 \mathrm{~m} / \mathrm{c}, a$ второй одновременно бросают под углом $\alpha=30^{\circ} \kappa$ горизонту по направлению $\kappa$ первому камню со скоростью $v_{20}=8 \mathrm{~m} / \mathrm{c}$. Чему равно наименьшее расстояние между камнями в прочессе движения?

Решение. Перейдем в СО первого камня. Ускорение второго камня в этой СО находим по формуле (3):

$$
\vec{a}_{21}=\vec{a}_{2}-\vec{a}_{1}=\vec{g}-\vec{g}=0 .
$$

Значит, движение второго камня в СО первого камня происходит равномерно и прямолинейно со скоростью

$$
\vec{v}_{21}=\vec{v}_{20}-\vec{v}_{10}=\vec{v}_{20}+\left(-\vec{v}_{10}\right) .
$$

(Это свойство относительного движения иногда называют принципом барона Мюнхгаузена. Барон, как известно, любил кататься верхом на пушечном ядре.) Линия движения второго тела в СО первого тела построена на рисунке 11. Наименьшее расстояние между

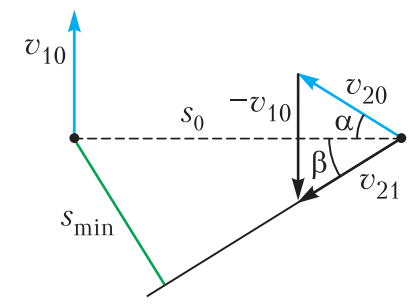

Рис. 11

камнями равно, как и в предыдущей задаче, длине перпендикуляра:

$$
s_{\text {min }}=s_{0} \sin \beta .
$$

А вот с нахождением синуса угла $\beta$ придется повозиться:

$$
\sin \beta=\frac{\left|v_{10}-v_{20} \sin \alpha\right|}{\sqrt{\left(v_{10}-v_{20} \sin \alpha\right)^{2}+\left(v_{20} \cos \alpha\right)^{2}}} .
$$

Подставляя числа, получаем

$$
\sin \beta=\frac{1}{7} \text {, и } s_{\min }=6 \text { м. }
$$

Принцип барона Мюнхгаузена используется и в следующей задаче.
Задача 15. Из некоторой точки одновременно бросают два камня: один в северном направлении под углом $\alpha_{1}=60^{\circ} \kappa$ горизонту со скоростью $v_{0}=18 \mathrm{~m} / \mathrm{c}$, другой в южном направлении под углом $\alpha_{2}=60^{\circ} \kappa$ горизонту со скоростью $v_{20}=48 \mathrm{~m} / \mathrm{c}$. Найдите расстояние между камнями через $t=$ $=1,5 \mathrm{c}$.

Решение. Перейдем в СО первого камня. Поскольку относительное ускорение равно нулю:

$$
\vec{a}_{21}=\vec{g}-\vec{g}=0,
$$

то в этой СО второй камень удаляется от первого равномерно и прямолинейно со скоростью

$$
\vec{v}_{21}=\vec{v}_{20}-\vec{v}_{10}
$$

а расстояние между камнями через время $t$ оказывается равным $s=v_{21} t$. Модуль относительной скорости найдем с помощью теоремы косинусов (против относительной скорости лежит угол $\beta=180^{\circ}-\alpha_{1}-\alpha_{2}=60^{\circ}$;

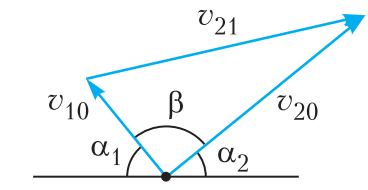

Pис. 12

рис.12):

$$
v_{21}=\sqrt{v_{20}^{2}+v_{10}^{2}-2 v_{20} v_{10} \cos \beta}=42 \mathrm{M} / \mathrm{c} .
$$

Тогда

$$
s=v_{21} t=63 \mathrm{M} .
$$

Конечно, эту задачу можно решить «в лоб», записав координаты камней через время $t$ и найдя расстояние между этими точками. Но в нашем подходе важно понимание, что ответ зависит не от углов $\alpha_{1}$ и $\alpha_{2}$, а от угла $\beta$. Если изменить условие, задав, например, $\alpha_{1}=50^{\circ}$ и $\alpha_{2}=70^{\circ}$, то решение $\ll$ в лоб» сильно усложнится (придется повозиться с тригонометрией), а в нашем подходе сразу видно, что ответ не изменится.

\section{Упражнения}

1. Эскалатор метрополитена, двигаясь равномерно, поднимает неподвижно стоящего на нем пассажира в течение одной минуты. По неподвижному эскалатору пассажир, двигаясь равномерно, поднимается за три минуты. Сколько секунд будет подниматься пассажир по движущемуся вверх эскалатору? 
2. В безветренную погоду для перелета из

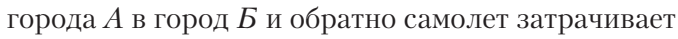
8 часов полетного времени. На сколько минут увеличится это время, если во время полета будет дуть ветер со скоростью $20 \mathrm{~m} / \mathrm{c}$ в направлении от $A$ к $Б$ ? Скорость самолета относительно воздуха $312 \mathrm{\kappa м} /$ ч .

3. Из пункта $A$ по взаимно перпендикулярным дорогам одновременно выехали два автомобиля: один со скоростью 80 км/ч , другой со скоростью 60 км/ч . С какой скоростью (в км/ч) они удаляются друг от друга?

4. При скорости ветра 20 м/с скорость капель дождя 40 м/с . Какой будет скорость капель при скорости ветра $5 \mathrm{~m} / \mathrm{c}$ ?

5. Самолет, совершающий перелеты из города $A$ в город $E$ и обратно, развивает в полете скорость 328 км/ч относительно воздуха. При боковом ветре, перпендикулярном линии полета, перелет туда и обратно занял 6 часов полетного времени. На сколько минут больше займет этот перелет, если ветер будет все время дуть в направлении от $A$ к $D$ ? Скорость ветра в обоих случаях $20 \mathrm{~m} / \mathrm{c}$.

6. При переправе через реку шириной 60 м надо попасть в точку, лежащую на 80 м ниже по течению, чем точка старта. Лодочник управляет моторной лодкой так, что она движется точно к цели со скоростью 8 м/с относительно берега. Какова при этом скорость лодки относительно воды, если скорость течения реки $2,8 \mathrm{~m} / \mathrm{c}$ ?

7. Когда пассажиру осталось дойти до двери вагона 25 м, поезд тронулся с места и стал разгоняться с ускорением $0,5 \mathrm{~m} / \mathrm{c}^{2}$. Пассажир побежал с постоянной скоростью. При какой минимальной скорости он догонит свой вагон?

8. Из одной точки одновременно бросают два тела: одно горизонтально со скоростью 6 м/с , другое вертикально со скоростью 8 м/с . На каком расстоянии друг от друга будут находиться тела через 2 c?

9. Два камня расположены на одной горизонтали на расстоянии 30 м друг от друга. Один камень бросают вертикально вверх со скоростью 9 м/с, а второй одновременно бросают горизонтально по направлению к первому камню со скоростью $12 \mathrm{~m} / \mathrm{c}$. Чему равно наименьшее расстояние между камнями в процессе движения?

10. Через блок перекинули нерастяжимую нить, к концам которой прикрепили два груза. В некоторый момент ось блока поднимается вертикально вверх со скоростью $2 \mathrm{~m} / \mathrm{c}$, а один из грузов опускается со скоростью 3 м/с . С какой скоростью движется в этот момент другой груз?

\section{О Л И М П И А Д Ы}

\section{Муниципальный этап LIII Всероссийской олимпиады школьников по физике}

\section{ЗАДАЧИ ОЛИМПИАДЫ}

\section{7 класс}

\section{Задача 1. Забывчивый Баг}

Теоретик Баг измерил массу и объем кирпича. Они оказались равны, соответственно, $m=5400(\ldots)$ и $V=1800$ (..). Затем он вычислил плотность кирпича $\rho=0,000000003$ (...). Однако Баг забыл указать, в каких единицах записаны эти величины. Приведя рассуждения, основанные на вашем жизненном опыте, восстановите единицы, в которых выражены масса, объем и плотность кирпича. Известно, что масса измеряется в граммах, килограммах или тоннах, объем измеряется в мм $^{3}, \mathrm{~cm}^{3}$, дм ${ }^{3}$ или $\mathrm{m}^{3}$.

\section{С.Кармазин}

\section{Задача 2. Два участка пути}

На первом участке дороги автомобиль ехал со скоростью $v_{1}=45 \mathrm{\kappa m} / 4$, на втором - со скоростью $v_{2}=60 \mathrm{\kappa м} / ч$. Средняя скорость движения на всем пути оказалась равной $v_{\text {ср }}=50$ км/ч. Какой из участков длиннее и во сколько раз?

\section{В.Слободянин}

\section{Задача 3. На карусели}

Экспериментатор Глюк установил, что он совершает полный круг, проходя по краю 